\title{
A Web Services Method on Embedded Systems
}

\author{
Whe Dar Lin \\ The Overseas Chinese Institute of Technology, Dept of Information Management, \\ No. 100, Chiao Kwang Road, Taichung 40721, Taiwan \\ darlin@ocit.edu.tw
}

\begin{abstract}
An embedded system is driven by the rapid growth of the Internet, communication technologies, pervasive computing, and portable consumer electronics. This paper describes how mobile-based services on embedded systems of Web services can be implemented on low cost ARM microprocessors with reasonable performance. ARM is a high-performance, low-cost, lowpower RISC processor. A great deal of attention has focused on simple step. We design a new method using time from which a new required was generated by the home mobile-based server and the visiting network to translate method to protocol in the mobile agents for embedded systems. We can implement mobile devices in mobile-based services where home mobile-based server must reliability connection each other. For our mobile-based service playing an increasingly important role in enhance the reliability, we propose a framework to achieve property for mobile agents of Web services.
\end{abstract}

\section{Introduction}

In a mobile web service providing lots of advantages for platform independency, easy personal services communication system, the computation cost and the communication cost are the key factors when a protocol being designed on embedded systems for Web services application.[1]

Web services consist of XML-based system, component based distributed computing technology independent of Web services. Let $\mathrm{K}_{\mathrm{h}, \mathrm{v}}$ be the secret key shared by $\mathrm{H}$ and $V$. Let $K_{h, i}$ be the secret key shared by $H$ and $M\left(\operatorname{ID}_{i}\right)$. The $\{m\}_{\text {eh }}$ denotes the ciphertext of $\mathrm{m}$ encrypted by some public key cryptosystem using public key $\mathrm{e}_{\mathrm{h}}$. The $(\mathrm{m})_{\mathrm{k}}$ denotes the ciphertext of $\mathrm{m}$ encrypted using the secret key $\mathrm{k}$ of some secure symmetric cryptosystem.

We consider a service network of $\mathrm{n}$ agents and an adaptive adversary that can corrupt up to a minority $\mathrm{n} / 2$ of mobile agents. The mobile agents have access to a broadcast channel, there are insecure links between each pairs of them. In our system, $\mathrm{H}$ has a public key $\mathrm{e}_{\mathrm{h}}$, and the corresponding secret keys $\mathrm{p}_{\mathrm{h}}$ and $\mathrm{q}_{\mathrm{h}}$, where $\mathrm{p}_{\mathrm{h}}$ and $\mathrm{q}_{\mathrm{h}}$ are two large strong primes, and $\mathrm{O}_{\mathrm{h}}=\mathrm{p}_{\mathrm{h}} * \mathrm{q}_{\mathrm{h}}$.

The system has a large prime $\mathrm{P}$, and $\mathrm{Q}$ is a prime factor of $\mathrm{P}-1$. Let $\mathrm{g}$ be an element of order $\mathrm{Q}$ in $\mathrm{Z}_{\mathrm{P}}{ }^{*}$. $\mathrm{H}$ has a secret key $\mathrm{x}_{\mathrm{h}}$ and a public key $\mathrm{y}_{\mathrm{h}}$. When mobile device $\mathrm{MD}$ wants to send a privacy message in the wireless network, $M$ must purchase a privacy ticket from $\mathrm{H}$. This is based on the bound for algebraic curve, the number of point of a curve of $g$ over a finite field for large enough $\mathrm{g}$. To evaluate the proportion of complexity analysis, we consider the number of point of over Fq which is approximatively q. The complexity of our method will be exponential in the size of q, so we will count the number of operations which can be done in polynomial time by addition and mul- 
tiplication and hashing, which can satisfy the requirement of low computational, and communication cost due to the limited power of handset application..

In our system, $M$ randomly selects an integers a and computes respectively $A=g^{a}$ mod P, A time stamp T, an expire time day $\mathrm{T}_{\text {expire }}$, and the certificate $\mathrm{Cert}_{\mathrm{i}}=\left(\mathrm{ID}_{\mathrm{i}}, \mathrm{A}\right.$, $\mathrm{T})_{\mathrm{Kh}, \mathrm{i}}$. $\mathrm{V}$ only transfers the received encrypted message send to $\mathrm{H}$. $\mathrm{H}$ receiving the message from the visit network, $H$ first decrypts the message and then checks if M's identification is valid by verifying if $\mathrm{T}$ is in the content of the certificate $\mathrm{Cert}_{\mathrm{i}}$ and if $\mathrm{T}$ has not been presented before.

$$
\mathrm{S}_{\mathrm{M}}=x_{h}+b^{*} B * A * T_{\text {expire }} \bmod P-1
$$

If yes, $\mathrm{H}$ computes, where $\mathrm{x}_{\mathrm{h}}$ is the secret key of home domain, $\mathrm{y}_{\mathrm{h}}\left(y_{h}=g^{x_{h}} \bmod P\right)$ is the public key of the home domain, a is a random number $\left(A=g^{a} \bmod P\right.$ ) and $\mathrm{b}$ is a random number $\left(B=g^{b} \bmod P\right)$. And then $\mathrm{H}$ deducts a fixed amount of money from M's account. Then he sends the privacy ticket $\left\{S_{M}\right\}_{K h, i}$ back to $V$. The mobile device can check the ticket and see if the following equation holds or not.

$$
\begin{aligned}
& g^{S_{M}}=y_{h} B^{B A T_{\text {expire }}} \bmod P \text { where } \\
& g^{S_{M}}=g^{x_{h}+b^{*} B^{*} A^{*} T_{\text {expire }}}=\left(g^{x_{h}}\right)\left(g^{b}\right)^{B A T_{\text {expire }}}=y_{h} B^{B A T_{\text {expire }}}
\end{aligned}
$$

If the above equation holds, the privacy information $\left(B, T_{\text {expire }}, S_{M}\right)$ is validly issued by $\mathrm{H}\left(\mathrm{H}\right.$ has secret key $\mathrm{x}_{\mathrm{h}}$ and public key $\left.\mathrm{y}_{\mathrm{h}}\right)$.

\subsection{Only One Step Service Method}

In the service phase, mobile device (MD) uses information $\left(B, T_{\text {expire }}, S_{M}\right)$ and sends his Name and time stamp $\mathrm{T}_{\text {now }}, \mathrm{T}_{\text {expire }}, \mathrm{K}$ and $\mathrm{L}$ to $\mathrm{V}$.

$\mathrm{MD}$ randomly selects random numbers $\mathrm{a}, \mathrm{c}$ and $\mathrm{y}$ then computes respectively $\mathrm{A}=\mathrm{g}^{\mathrm{a}}$ $\bmod \mathrm{P}, \mathrm{C}=\mathrm{g}^{\mathrm{c}} \bmod \mathrm{P}, \mathrm{Y}=\mathrm{C}^{\mathrm{y}} \bmod \mathrm{P}$ and $\mathrm{H}$ has public key $\mathrm{y}_{\mathrm{h}}$.

$\mathrm{T}_{\text {now }}$ is a real time stamp. The mobile unit computes Name as follows: Name = $\mathrm{y}_{\mathrm{h}}\|\mathrm{C}\| \mathrm{E}$ where $\mathrm{E}=\mathrm{A} * \mathrm{~B}$ mod $\mathrm{P}$. And $\mathrm{M}$ computes $\mathrm{L}$

$$
\mathrm{L}=\mathrm{S}_{\mathrm{M}}+\mathrm{a} * \mathrm{~T}_{\text {expire }}+\mathrm{c} * \mathrm{~K}+\mathrm{c} * \mathrm{k} * \mathrm{~T}_{\text {now }}
$$

$\mathrm{V}$ can verify (Name, $T_{\text {now }}, T_{\text {exp ire }}, K, L$ ) according to the equation (4)

$$
g^{L}=y_{h} B^{B A T_{\text {expire }}} A^{T_{\text {expire }}} C^{K} K^{T_{\text {now }}} \bmod P
$$

If the above equation holds, then the mobile device (MD) can use the mobile-based service ticket (Name, $\left.T_{\text {now }}, T_{\text {exp ire }}, K, L\right)$.

\subsection{Discussion}

Proposition: Our proposed protocol can support the Web-based services on mobile EC location.

Proof: Let $\mathrm{N}$ be a random variable with probability distribution $\mathrm{P}$ and $\mathrm{N}>0$, if $\mathrm{K}$ is large enough, then $\mathrm{N}^{\mathrm{K}}$ distributed according to independent realization of $\mathrm{N}$. More 
precisely, the probability of the typically event tends to zero faster than $1 / \mathrm{N}^{2}$. We know much weaker than uniformity condition. In the service phase, when MS visits $\mathrm{VN}$ nodes $\left(\mathrm{V}_{1}, \mathrm{~V}_{2}, \ldots, \mathrm{V}_{\mathrm{N}}\right)$, Home account management system deducts a fixed amount of money from MS's account, then Home account management agent will broadcasts $\left(\mathrm{B}, \mathrm{T}_{\text {expire }}, \mathrm{E}_{\mathrm{M}}\right)_{\mathrm{Kh}, \mathrm{i}}$ to $\mathrm{VN}$ nodes $\left(\mathrm{V}_{1}, \mathrm{~V}_{2}, \ldots, \mathrm{V}_{\mathrm{N}}\right)$, where $\mathrm{K}_{\mathrm{h}, \mathrm{i}}$ is the secret key shared with $\mathrm{ID}_{\mathrm{i}}$ and home domain, $\mathrm{V}_{\mathrm{j}}$ is the visit domain ID number with mobile device $\mathrm{M}$ and home domain $\mathrm{H}$. When $\mathrm{VN}$ nodes $\left(\mathrm{V}_{1}, \mathrm{~V}_{2}, \ldots, \mathrm{V}_{\mathrm{N}}\right)$ receive the message $\left(\mathrm{B}, \mathrm{T}_{\text {expire}}\right.$, $\left.\mathrm{S}_{\mathrm{M}}\right)_{\mathrm{Kh}, \mathrm{i}}$. There is no information about user $\mathrm{ID}_{\mathrm{i}}$, the secret key $\mathrm{K}_{\mathrm{h}, \mathrm{i}}$ is only known to HMBS and user $I_{\mathrm{i}}$. No attacker can get $\mathrm{K}_{\mathrm{h}, \mathrm{i}}$ from $\left(\mathrm{B}, \mathrm{T}_{\text {expire }}, \mathrm{S}_{\mathrm{M}}\right)_{\mathrm{Kh}, \mathrm{i}}$ because assumed secure since it is infeasible. The attacker and $\mathrm{V}_{\mathrm{j}}$ do not know the cryptographic algorithm key. This is exactly the discrete logarithm problem so the intruder fails. In fact, our proposed scheme, user $\mathrm{ID}_{\mathrm{i}}$ is kept unknown to attacker and $\mathrm{V}_{\mathrm{j}}$. Therefore, the MS $\left(\mathrm{ID}_{\mathrm{i}}\right)$ can use Name $\mathrm{j}_{\mathrm{j}}$ to roam $\mathrm{VN}\left(\mathrm{V}_{\mathrm{j}}\right)$. The different IDs used in different VNs are that our proposed protocol keeps the Web-based services on mobile EC location. Since B is not uniformly distributed between zero and $2^{\mathrm{g}}$. We can provides a heuristic argument to show $\mathrm{P}=2^{\text {hg }}$. With $\mathrm{h}>1 / 2$, then with probability greater than $\log (\mathrm{h} / 1-\mathrm{h})$ for cover application in complexity is $2^{\mathrm{hg}+\mathrm{O}(1)}$.

\section{Conclusion}

In this paper, we propose an efficient general service method on embedded system for mobile-based services on Web-based system. Our approach of new scheme can adopt a chaining relationship to prevent some known attacks and provide data integrity and mobile agent. Mobile devices only compute addition, multiplication and hashing, which can satisfy the requirement of low computational, and communication cost due to the limited power of handset application. A great deal of attention has focused on simple step. We design a new method using time from which a new required was generated by the home mobile-based server and the visiting network to translate method to secure protocol in the mobile agents for embedded systems. Our method can face the rapid growth of the Internet, significant number of Web-based information processing has come to rely on services cluster technology to service lots of mobile users.

\section{References}

1. Whe Dar Lin, "EC Services Use Different Web-based systems," Lecture Notes in Computer Science, LNCS-2658, pp1059-1068, 2003. 